https://doi.org/10.15407/ujpe63.7.600

T. ABEBE, N. GEMECHU

Jimma University, Department of Physics

(P. O. Box 378, Jimma, Ethiopia; e-mail: tam1704@gmail.com)

\title{
TWO-LEVEL ATOM WITH SQUEEZED LIGHT FROM OPTICAL PARAMETRIC OSCILLATORS
}

\begin{abstract}
The dynamics of a coherently driven two-level atom with parametric amplifier and coupled to a vacuum reservoir is analyzed. The combination of the master equation and the quantum Langevin equation is presented to study the quantum properties of light. By using these equations, we have determined the time evolution of the expectation values of the cavity mode and atomic operators. Moreover, with the aid of these results, the correlation properties of noise operators, and the large-time approximation scheme, we calculate the mean photon number, power spectrum, second-order correlation function, and quadrature variances for the cavitymode light and fluorescence. It is found that the half-width of the power spectrum for the fluorescent light in the presence of a parametric amplifier increases, while it decreases for the cavity-mode light. Moreover, we have found the probability for the atom to be in the upper level in the presence of a parametric amplifier.
\end{abstract}

Keywords: power spectrum, quadrature squeezing, second-order correlations.

\section{Introduction}

The interaction of radiation with atoms is one of the central problems in quantum optics. This kind of interaction is involved in various physical processes of interest such as resonance fluorescence and laser dynamics. In addition, the interaction of twolevel atoms with squeezed light has been extensively studied, as it exhibits interesting nonclassical features. Moreover, the effect of the squeezed light on the properties of the fluorescent light emitted by the two-level atom has attracted a great deal of interest [1-11]. In this respect, a two-level atom embedded in a broadband squeezed vacuum has studied by Gardiner [1], and it was found that the atomic polarization quadratures are phase-dependent. Moreover, in the case where the atom is immersed in a broadband squeezed vacuum and driven by coherent light, the fluorescence spectrum was studied by Carmichael et al. [2].

The properties of the fluorescent light emitted by a two-level atom in a cavity driven by coherent light and coupled to a squeezed vacuum have been studied by several authors [3-12]. Some of these studies have shown that the width of the incoherent spec-

(C) T. ABEBE, N. GEMECHU, 2018 trum of the fluorescent light has been modified by the squeezed light. Furthermore, the composite system consisting of a two-level atom and a parametric amplifier has been considered by various authors. In addition, Jin and Xiao [13] showed that the presence of two-level atoms increases considerably the degree of squeezing of the light produced by a parametric oscillator. Moreover, Eyob [14] studied a coherently driven two-level atom inside a parametric oscillator operating below the threshold. He found that the presence of a parametric amplifier leads to an increase in the width of the power spectrum of the fluorescent light in the weak and strong driving light limits, and the effect of the presence of a parametric amplifier on the second-order correlation function is to enhance its decay rate.

In this paper, we will consider a degenerate parametric oscillator, whose cavity contains a two-level atom coupled with a vacuum reservoir.

In an optical parametric amplifier, the high frequency is called the pump, the lower frequency of primary interest is called the signal, and the remaining frequency is called the idler [15]. The interaction of the signal light, produced by a parametric amplifier, with the two-level atom leads to a generation of the fluorescent light. Thus, the cavity mode in this case consists of the signal light and the fluorescent light

ISSN 2071-0194. Ukr. J. Phys. 2018. Vol. 63, No. 7 
emitted by the two-level atom. Conversely, an optical parametric oscillator operating below the threshold is a typical source of the squeezed light. It is then quite interesting to study the effect of the squeezed light from a parametric oscillator on the quantum properties of the fluorescent light emitted by the two-level atom. We derive the equations of evolution for the expectation values of atomic and cavity mode operators, by using the master and quantum Langevin equations in the bad-cavity limit. Applying the resulting equations, we calculate the power spectrum and the second-order correlation function of the $\mathrm{flu}$ orescent light. Moreover, we determine the the mean photon number and the quadrature variance for cavity and fluorescence lights.

\section{Equations of Evolution of Cavity Mode and Atomic Expectation Values}

A single two-level atom inside a degenerate parametric oscillator coupled to a vacuum reservoir is considered (see Fig. 1). As is clearly indicated, the upper and lower levels of the atom are represented by $|a\rangle$ and $|b\rangle$. The cavity mode and the atomic transition are assumed to be at resonance. In a degenerate parametric oscillator, a pump photon of frequency $2 \omega$ is down converted into a pair of highly correlated signal photons each of frequency $\omega[16]$. It turns out that the signal light is in a squeezed state. With the pump mode treated classically, the parametric interaction and the interaction of the two-level atom with the cavity mode can be described by the Hamiltonian

$\hat{H}=\frac{i \varepsilon}{2}\left(\hat{a}^{\dagger 2}-\hat{a}^{2}\right)+i g\left[\hat{\sigma}_{+} \hat{a}-\hat{a}^{\dagger} \hat{\sigma}_{-}\right]$,

in which $\varepsilon$, assumed to be real and constant, is proportional to the amplitude of the pump mode that drives the NLC (nonlinear cavity), $\hat{a}$ and $\hat{a}^{\dagger}$ are, respectively, the creation and annihilation operators for the cavity mode, $g$ is the atom-cavity mode coupling constant, and $\hat{\sigma}_{+}=|a\rangle\langle b|$ and $\hat{\sigma}_{-}=|b\rangle\langle a|$ are atomic operators satisfying the commutation relations $\left[\hat{\sigma}_{ \pm}, \hat{\sigma}_{\mp}\right]= \pm \hat{\sigma}_{z},\left[\hat{\sigma}_{ \pm}, \hat{\sigma}_{z}\right]=\mp 2 \hat{\sigma}_{ \pm},\left[\hat{\sigma}_{z}, \hat{\sigma}_{ \pm}\right]=$ $= \pm 2 \hat{\sigma}_{ \pm}$with $\hat{\sigma}_{z}=|a\rangle\langle a|-| b\rangle\langle b|$.

In studying the nonclassical features and the quantum properties of the generated radiation, it is essential to derive various correlations from the master equation. For the system under consideration, the master equation is obtained by substituting Eq. (1)
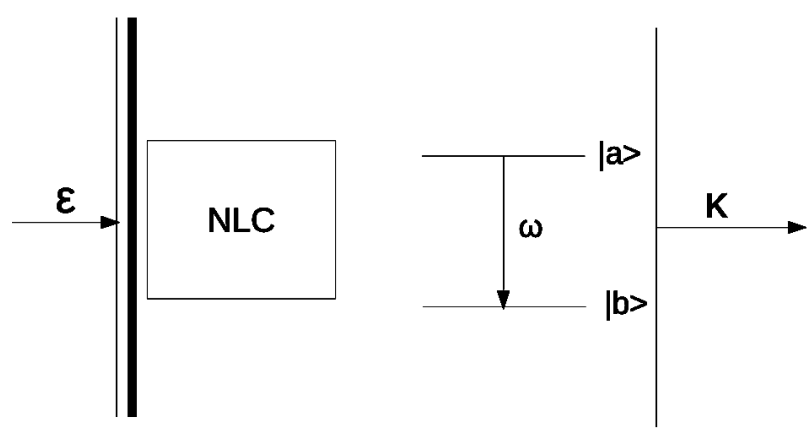

$a$

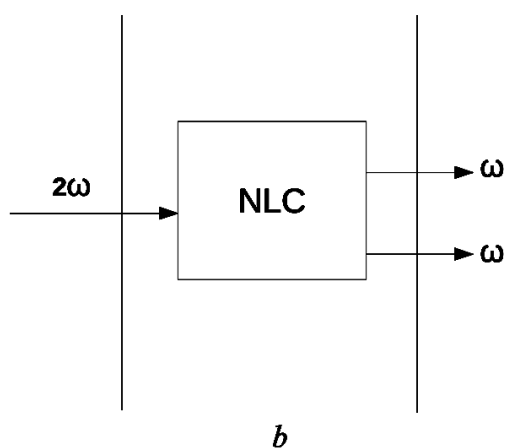

Fig. 1. Scheme of a single two-level atom inside a parametric oscillator coupled with a vacuum reservoir $(a)$. Degenerate parametric oscillator $(b)$

into the relation $[16]$

$\frac{d}{d t} \hat{\rho}(t)=[\hat{H}, \hat{\rho}]+\frac{\kappa}{2}\left[2 \hat{a} \hat{\rho} \hat{a}^{\dagger}-\hat{a}^{\dagger} \hat{a} \hat{\rho}-\hat{\rho} \hat{a}^{\dagger} \hat{a}\right]$.

We get

$\frac{d}{d t} \hat{\rho}(t)=g\left[\hat{\sigma}_{+} \hat{a} \hat{\rho}-\hat{\rho} \hat{\sigma}_{+} \hat{a}-\hat{a}^{\dagger} \hat{\sigma}_{-} \hat{\rho}+\hat{\rho} \hat{a}^{\dagger} \hat{\sigma}_{-}\right]+$

$+\frac{\varepsilon}{2}\left[\hat{a}^{\dagger 2} \hat{\rho}-\hat{\rho} \hat{a}^{\dagger 2}-\hat{a}^{2} \hat{\rho}+\hat{\rho} \hat{a}^{2}\right]+$

$+\frac{\kappa}{2}\left[2 \hat{a} \hat{\rho} \hat{a}^{\dagger}-\hat{a}^{\dagger} \hat{a} \hat{\rho}-\hat{\rho} \hat{a}^{\dagger} \hat{a}\right]$

where $\kappa$ is a cavity damping constant. It is possible to obtain the equation of evolution for the cavity mode and atomic operators. To this end, applying Eq. (3) and the fact that

$\frac{d}{d t}\langle\hat{O}\rangle=\operatorname{Tr}\left(\frac{d \hat{\rho}}{d t} \hat{O}\right)$

we can readily obtain

$\frac{d}{d t}\left\langle\hat{\sigma}_{-}\right\rangle=-g\left\langle\hat{\sigma}_{z} \hat{a}\right\rangle$ 


$$
\begin{aligned}
& \frac{d}{d t}\left\langle\hat{\sigma}_{z}\right\rangle=2 g\left(\left\langle\hat{a}^{\dagger} \hat{\sigma}_{-}\right\rangle+\left\langle\hat{\sigma}_{+} \hat{a}\right\rangle\right), \\
& \frac{d}{d t}\langle\hat{a}\rangle=-\frac{\kappa}{2}\langle\hat{a}\rangle+\varepsilon\left\langle\hat{a}^{\dagger}\right\rangle-g\left\langle\hat{\sigma}_{-}\right\rangle, \\
& \frac{d}{d t}\left\langle\hat{a}^{2}\right\rangle=-\kappa\left\langle\hat{a}^{2}\right\rangle+2 \varepsilon\left\langle\hat{a}^{\dagger} \hat{a}\right\rangle e+\frac{\gamma_{c} \varepsilon / \kappa}{1-4 \varepsilon^{2} / \kappa^{2}}+ \\
& +\frac{2 \gamma_{c} \varepsilon / \kappa}{\left(1-4 \varepsilon^{2} / \kappa^{2}\right)^{2}}\left\langle\hat{\sigma}_{z}\right\rangle+\varepsilon, \\
& \frac{d}{d t}\left\langle\hat{a}^{\dagger} \hat{a}\right\rangle=-\kappa\left\langle\hat{a}^{\dagger} \hat{a}\right\rangle-\frac{\gamma_{c}}{1-4 \varepsilon^{2} / \kappa^{2}}\left\langle\hat{\sigma}_{+} \hat{\sigma}_{-}\right\rangle+ \\
& +\varepsilon\left(\left\langle\hat{a}^{2}\right\rangle+\left\langle\hat{a}^{\dagger 2}\right\rangle\right)-\frac{2 \gamma_{c} \varepsilon^{2} / \kappa}{\left(1-4 \varepsilon^{2} / \kappa^{2}\right)^{2}}\left\langle\hat{\sigma}_{z}\right\rangle,
\end{aligned}
$$

where $\gamma_{c}=4 g^{2} / \kappa$ is the stimulated emission decay constant. On the basis of Eq. (7), we can write

$$
\frac{d \hat{a}}{d t}=-\frac{\kappa}{2} \hat{a}+\varepsilon \hat{a}^{\dagger}-g \hat{\sigma}_{-}+\hat{F},
$$

where $\hat{F}$ is a noise operator associated with a vacuum reservoir and having the following correlation properties:

$$
\begin{aligned}
& \langle\hat{F}(t)\rangle=0, \\
& \left\langle\hat{F}^{\dagger}(t) \hat{F}\left(t^{\prime}\right)\right\rangle=0, \\
& \left\langle\hat{F}(t) \hat{F}^{\dagger}\left(t^{\prime}\right)\right\rangle=\kappa \delta\left(t-t^{\prime}\right), \\
& \left\langle\hat{F}^{\dagger}(t) \hat{F}^{\dagger}\left(t^{\prime}\right)\right\rangle=\left\langle\hat{F}(t) \hat{F}\left(t^{\prime}\right)\right\rangle=0 .
\end{aligned}
$$

Equations (11)-(14) represent the mean and correlation properties of the noise operator.

It can be noted that Eqs. (5)-(9) are nonlinear coupled differential equations. Therefore, it is impossible to obtain exact solutions. Then one can obtain the approximate solutions of these equations in the badcavity limit. In this limit, the cavity damping constant is much greater than the cavity atomic decay rate $\left(\kappa \gg \gamma_{c}\right)$, and the cavity mode variables decay faster than the atomic variables. It is possible to set the time derivatives of the cavity mode variables equal to zero, while keeping the zero-order atomic and cavity mode variables at time $t$. In view of this, Eq. (10) yields

$$
\begin{aligned}
& \hat{a}(t)=-\frac{2 g / \kappa}{1-4 \varepsilon^{2} / \kappa^{2}} \hat{\sigma}_{-}(t)-\frac{4 g \varepsilon / \kappa^{2}}{1-4 \varepsilon^{2} / \kappa^{2}} \hat{\sigma}_{+}(t)+ \\
& +\frac{4 / \kappa^{2}}{1-4 \varepsilon^{2} / \kappa^{2}}\left[\frac{\kappa}{2} \hat{F}(t)+\varepsilon \hat{F}^{\dagger}\right] .
\end{aligned}
$$

By substituting this result into (5) and (6) and taking the expectation value of the resulting equations, one can easily establish that

$$
\begin{aligned}
& \frac{d}{d t}\left\langle\hat{\sigma}_{-}(t)\right\rangle=-\frac{\xi}{2}\left\langle\hat{\sigma}_{-}(t)\right\rangle+\frac{\xi \varepsilon}{\kappa}\left\langle\hat{\sigma}_{+}(t)\right\rangle-\frac{4 g / \kappa^{2}}{1-4 \varepsilon^{2} / \kappa^{2}} \times \\
& \times\left[\frac{\kappa}{2}\left\langle\hat{F}(t) \hat{\sigma}_{z}(t)\right\rangle+\varepsilon\left\langle\hat{\sigma}_{z}(t) \hat{F}^{\dagger}(t)\right\rangle\right], \\
& \frac{d}{d t}\left\langle\hat{\sigma}_{z}(t)\right\rangle=-2 \xi\left\langle\hat{\sigma}_{+}(t) \hat{\sigma}_{-}(t)\right\rangle+\frac{8 g / \kappa^{2}}{1-4 \varepsilon^{2} / \kappa^{2}} \times \\
& \times\left[\frac{\kappa}{2}\left(\left\langle\hat{F}^{\dagger}(t) \hat{\sigma}_{-}(t)\right\rangle+\left\langle\hat{\sigma}_{+}(t) \hat{F}(t)\right\rangle\right)+\right. \\
& \left.+\varepsilon\left(\left\langle\hat{F}(t) \hat{\sigma}_{-}(t)\right\rangle+\left\langle\hat{\sigma}_{+}(t) \hat{F}^{\dagger}(t)\right\rangle\right)\right], \\
& \text { where } \gamma_{c} \\
& \xi=\frac{17)}{1-4 \varepsilon^{2} / \kappa^{2}} .
\end{aligned}
$$

Equation (16) has a well-behaved solution provided that $\xi>0$, if $\varepsilon / \kappa<1 / 2$. Then it is easily seen that the threshold condition for the parametric oscillator is $\varepsilon / \kappa=1 / 2$. The correlation properties between a noise operator and an atomic operator that appear in Eqs. (16) and (17) are found to be

$$
\begin{aligned}
& \left\langle\hat{F}(t) \hat{\sigma}_{-}(t)\right\rangle=\left\langle\hat{\sigma}_{+}(t) \hat{F}^{\dagger}(t)\right\rangle=-\frac{(2 g \varepsilon / \kappa)\left\langle\hat{\sigma}_{z}(t)\right\rangle}{1-4 \varepsilon^{2} / \kappa^{2}}, \\
& \left\langle\hat{\sigma}_{-}(t) \hat{F}^{\dagger}(t)\right\rangle=\left\langle\hat{F}(t) \hat{\sigma}_{+}(t)\right\rangle=-\frac{g\left\langle\hat{\sigma}_{z}(t)\right\rangle}{1-4 \varepsilon^{2} / \kappa^{2}}, \\
& \left\langle\hat{\sigma}_{z}(t) \hat{F}^{\dagger}(t)\right\rangle=\frac{4 g / \kappa}{1-4 \varepsilon^{2} / \kappa^{2}}\left[\frac{\kappa}{2}\left\langle\hat{\sigma}_{+}(t)\right\rangle+\varepsilon\left\langle\hat{\sigma}_{-}(t)\right\rangle\right], \\
& \left\langle\hat{F}^{\dagger}(t) \hat{\sigma}_{-}(t)\right\rangle=\left\langle\hat{\sigma}_{+}(t) \hat{F}(t)\right\rangle=0, \\
& \left\langle\hat{\sigma}_{z}(t) \hat{F}(t)\right\rangle=0 .
\end{aligned}
$$

Using the relation $\left\langle\hat{\sigma}_{+} \hat{\sigma}_{-}\right\rangle=\left(\left\langle\hat{\sigma}_{z}\right\rangle+1\right) / 2$ and in view of the fact that $\left\langle\hat{\sigma}_{-}\right\rangle^{*}=\left\langle\hat{\sigma}_{+}\right\rangle$and $\left\langle\hat{\sigma}_{z}\right\rangle^{*}=\left\langle\hat{\sigma}_{z}\right\rangle$, it can be verified that

$$
\begin{aligned}
\frac{d}{d t}\left\langle\hat{\sigma}_{-}(t)\right\rangle & =-\frac{\Gamma}{2}\left\langle\hat{\sigma}_{-}(t)\right\rangle+\frac{\varepsilon \Gamma}{\kappa}\left\langle\hat{\sigma}_{+}(t)\right\rangle, \\
\frac{d}{d t}\left\langle\hat{\sigma}_{+}(t)\right\rangle & =-\frac{\Gamma}{2}\left\langle\hat{\sigma}_{+}(t)\right\rangle+\frac{\varepsilon \Gamma}{\kappa}\left\langle\hat{\sigma}_{-}(t)\right\rangle, \\
\frac{d}{d t}\left\langle\hat{\sigma}_{z}(t)\right\rangle & =-\Gamma\left\langle\hat{\sigma}_{z}(t)\right\rangle-\xi,
\end{aligned}
$$

in which

$\Gamma=\frac{\gamma_{c}\left(1+4 \varepsilon^{2} / \kappa^{2}\right)}{\left(1-4 \varepsilon^{2} / \kappa^{2}\right)^{2}}$

is the cavity atomic decay rate, and $\gamma_{c}=4 g^{2} / \kappa$ is the stimulated emission decay constant in the absence of a nonlinear crystal, $\varepsilon=0$. Moreover, the presence of a nonlinear crystal enhances the cavity atomic decay rate from $\gamma_{c}$ to $\Gamma$.

ISSN 2071-0194. Ukr. J. Phys. 2018. Vol. 63, No. 7 


\section{Power Spectrum}

In this section, the analysis of the power spectrum of the fluorescent light and the cavity light are presented.

\subsection{The power spectrum of the fluorescent light}

The power spectrum of the fluorescent light can be expressed in terms of the atomic operators as

$S(\omega)=2 \operatorname{Re} \int_{0}^{\infty} e^{i \omega \tau}\left\langle\hat{\sigma}_{+}(t) \hat{\sigma}_{-}(t+\tau)\right\rangle_{s s} d \tau$.

To obtain the value $\left\langle\hat{\sigma}_{+}(t) \hat{\sigma}_{-}(t+\tau)\right\rangle_{s s}$ expressed in Eq. (28), it is necessary to introduce the new variables

$\gamma_{ \pm}=\left\langle\hat{\sigma}_{+}(t)\right\rangle \pm\left\langle\hat{\sigma}_{-}(t\rangle\right.$.

One can easily show that

$\frac{d}{d t} \gamma_{ \pm}=-\lambda_{ \pm} \gamma_{ \pm}$

where $\lambda_{ \pm}=\Gamma\left(\frac{1}{2} \pm \frac{\varepsilon}{\kappa}\right)$. The formal solution of Eq. (30) can be written as

$\gamma_{ \pm}(t+\tau)=\gamma_{ \pm}(t) e^{-\lambda_{ \pm} \tau}$

It then follows that

$\gamma_{+}(t+\tau)+\gamma_{+}(t+\tau)=\gamma_{+}(t) e^{-\lambda_{+} \tau}+\gamma_{-}(t) e^{-\lambda_{-} \tau}$.

In view of Eq. (29), one can write Eq. (32) as

$\left\langle\sigma_{-}(t+\tau)\right\rangle=\frac{1}{2}\left(\left\langle\sigma_{-}(t)\right\rangle+\left\langle\sigma_{+}(t)\right\rangle\right)\left[e^{-\lambda_{+} \tau}+e^{-\lambda_{-} \tau}\right]$.

Applying the quantum regression theorem to Eq. (33), one obtains

$\left\langle\sigma_{+}(t) \sigma_{-}(t+\tau)\right\rangle=\frac{1}{2}\left\langle\sigma_{+}(t) \sigma_{-}(t)\right\rangle\left[e^{-\lambda_{+} \tau}+e^{-\lambda_{-} \tau}\right]$.

The two-time correlation function takes, at a steady state, the form

$\left\langle\sigma_{+}(t) \sigma_{-}(t+\tau)\right\rangle_{s s}=\frac{\Gamma-\xi}{4 \Gamma}\left[e^{-\lambda_{+} \tau}+e^{-\lambda_{-} \tau}\right]$,

in which

$\left\langle\sigma_{+}(t) \sigma_{-}(t)\right\rangle_{s s}=(\Gamma-\xi) / 2 \Gamma$.

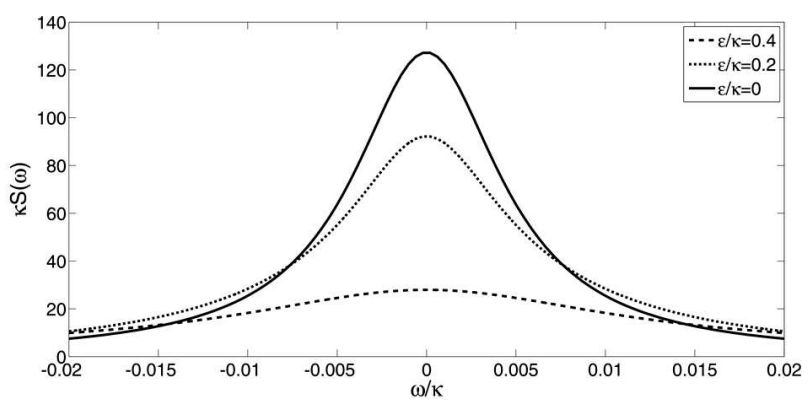

Fig. 2. Plots of the power spectrum of the fluorescent light versus $\omega / \kappa$ for different values of $\varepsilon / \kappa$

Substituting Eq. (35) into (28) and performing the integration, the power spectrum of the fluorescent light turns out to be

$S(\omega)=\left[\frac{\lambda_{+} / 2 \pi}{\lambda_{+}^{2}+\omega^{2}}+\frac{\lambda_{-} / 2 \pi}{\lambda_{-}^{2}+\omega^{2}}\right]$

It is clearly indicated by Eq. (37) that the halfwidths of the two Lorentzians are $\lambda_{+}$and $\lambda_{-}$. Thus, Fig. 2 shows that the power spectrum of the fluorescent light has a single peak centered at $\omega=0$. The power spectrum in the absence and presence of a parametric amplifier can be observed in Fig. 2. This figure shows that the presence of a parametric amplifier leads to an increase in the width of the spectrum. For instance, in the absence of a parametric amplifier, the half-width is found to be 0.005 , and it increases from 0.008 to 0.028 , when $\varepsilon / \kappa$ increases from 0.20 to 0.40 .

\subsection{Power spectrum of the cavity mode}

The power spectrum of the cavity mode can be expressed as

$P(\omega)=\frac{1}{\pi} \operatorname{Re} \int_{0}^{\infty} e^{i \omega \tau}\left\langle\hat{a}^{\dagger}(t) \hat{a}(t+\tau)\right\rangle_{s s} d \tau$.

Following a similar procedure that described for the power spectrum of the fluorescent light, the steadystate expectation value of the two-time correlation function that appears in Eq. (38) is found to be

$$
\begin{aligned}
& \left\langle\hat{a}^{\dagger}(t) \hat{a}(t+\tau)\right\rangle_{s s}=\bar{n}\left[\frac{e^{-\nu_{-} \tau}+e^{\nu_{+} \tau}}{2}\right]+ \\
& +\left\langle\hat{a}^{2}(t)\right\rangle_{s s}\left[\frac{e^{-\nu_{-} \tau}-e^{-\nu_{+} \tau}}{2}\right],
\end{aligned}
$$




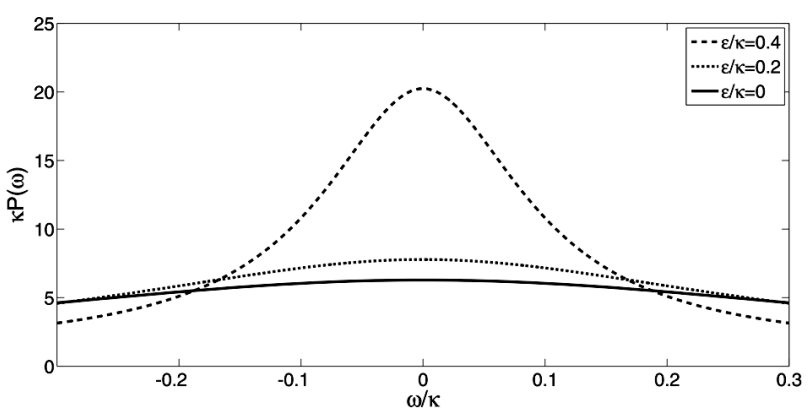

Fig. 3. Plots of the power spectrum of the cavity light versus $\omega / \kappa$ for different values of $\varepsilon / \kappa$

where $\nu_{ \pm}=\kappa\left(\frac{1}{2} \pm \frac{\varepsilon}{\kappa}\right)$. After performing the integration, it is possible to get

$$
\begin{aligned}
& P(\omega)=\left[\frac{\nu_{+} / 2 \pi}{\nu_{+}^{2}+\omega^{2}}+\frac{\nu_{-} / 2 \pi}{\nu_{-}^{2}+\omega^{2}}+\frac{(\kappa / 2 \varepsilon)\left(\nu_{-} / 2 \pi\right)}{\kappa^{2} \nu_{-}^{2}+\omega^{2}}-\right. \\
& \left.-\frac{(\kappa / 2 \varepsilon)\left(\nu_{+} / 2 \pi\right)}{\kappa^{2} \nu_{+}^{2}+\omega^{2}}\right] .
\end{aligned}
$$

Since the expression for the spectrum does not contain $\gamma_{c}$, the presence of a two-level atom does not affect the width of this spectrum. In Fig. 3, the power spectrum is shown in the absence and presence of a parametric amplifier. In this figure, the plots present the power spectrum of the cavity mode versus $\omega / \kappa$ for different values of $\varepsilon / \kappa$. It is found that the presence of a parametric amplifier decreases the width of the power spectrum, as $\varepsilon / \kappa$ increases. Moreover, comparing the results shown in Figs. 2 and 3 indicates that the half-width of the power spectrum of the fluorescent light in the presence of a parametric amplifier increases, while it decreses for the cavity light.

\section{Normalized Second-Order}

\section{Correlation Function of the Fluorescent Light}

In this section, the effect of a parametric amplifier on the intensity-intensity correlation function of the fluorescent light is determined. The normalized secondorder correlation function can be expressed in terms of the atomic operators as

$g^{(2)}(\tau)=\frac{\left\langle\hat{\sigma}_{+}(t) \hat{\sigma}_{+}(t+\tau) \hat{\sigma}_{-}(t+\tau) \hat{\sigma}_{-}(t)\right\rangle}{\left\langle\hat{\sigma}_{+}(t) \hat{\sigma}_{-}(t)\right\rangle}$.

We recall that

$$
\left\langle\hat{\sigma}_{+}(t+\tau) \hat{\sigma}_{-}(t+\tau)\right\rangle=\left(\left\langle\hat{\sigma}_{z}(t+\tau)\right\rangle+1\right) / 2 .
$$

604
Taking the formal solution of this expression and performing the integration, one can readily show that

$$
\left\langle\hat{\sigma}_{z}(t)\right\rangle=\left\langle\hat{\sigma}_{z}(0)\right\rangle e^{-\Gamma t}-\frac{\xi}{\Gamma}\left(1-e^{-\Gamma t}\right)
$$

Hence, making use of Eq. (43), it is possible to express $\left\langle\hat{\sigma}_{+}(t+\tau) \hat{\sigma}_{-}(t+\tau)\right\rangle=\left\langle\hat{\sigma}_{+}(t) \hat{\sigma}_{-}(t)\right\rangle e^{-\Gamma t}-$ $-\frac{\Gamma-\xi}{2 \Gamma}\left(1-e^{-\Gamma t}\right)$

Applying the quantum regression theorem to Eq. (44), one can write

$$
\begin{aligned}
& \left\langle\hat{\sigma}_{+}(t) \hat{\sigma}_{+}(t+\tau) \hat{\sigma}_{-}(t+\tau) \hat{\sigma}_{-}(t)\right\rangle= \\
& =\frac{\Gamma-\xi}{2 \Gamma}\left\langle\hat{\sigma}_{+}(t) \hat{\sigma}_{-}(t)\right\rangle\left(1-e^{-\Gamma t}\right) .
\end{aligned}
$$

In view of this result and Eq. (36), the second-order correlation function takes, in a steady state, the form

$g^{(2)}(\tau)=1-e^{-\Gamma \tau}$

It is of interest to analyze the second-order correlation function in the presence and absence of a parametric amplifier, as it exhibits different features in each case. One can observe from the above equation that $g^{(2)}(0)=0$ and, for $\tau>0, g^{(2)}(\tau)>0$. Therefore, for $\tau>0$, the second-order correlation function $g^{(2)}(\tau)>g^{(2)}(0)$. This shows that the fluorescent light exhibits the phenomenon of photon antibunching. This is due to the fact that a two-level atom cannot emit two or more photons simultaneously. After each emission, the atom returns to the lower level and must absorb a photon before another emission can take place. Moreover, the photons have a tendency to arrive at a detector separately rather than in pair. It can also be seen from Fig. 4 that, for relatively small values of $\tau$, the second-order correlation function is less than unity, which reflects the nonclassical feature of antibunching. One can also observe that, as $\varepsilon / \kappa$ increases, $g^{(2)}(\tau)$ approaches unity at a faster rate. It is also interesting to consider the dynamics of the two-level atom. Moreover, it is not difficult to realize that the second-order correlation function approaches unity as the degree of squeezing increases. The fact that $g^{(2)}(\tau)$ is less than unity reflects the nonclassical feature of antibunching.

ISSN 2071-0194. Ukr. J. Phys. 2018. Vol. 63, No. 7 
On the other hand, it is also interesting to study the dynamics of the two-level atom. Thus, by replacing $t+\tau$ by $t$ and $t$ by 0 in Eq. (44), we get

$\left\langle\hat{\sigma}_{+}(t) \hat{\sigma}_{-}(t)\right\rangle=\left\langle\hat{\sigma}_{+}(0) \hat{\sigma}_{-}(0)\right\rangle e^{-\Gamma t}-$

$-\frac{\Gamma-\xi}{2 \Gamma}\left(1-e^{-\Gamma t}\right)$.

Employing the relations

$\left(\left\langle\hat{\sigma}_{z}(0)\right\rangle+1\right) / 2=\left\langle\hat{\sigma}_{+}(0) \hat{\sigma}_{-}(0)\right\rangle$

and

$\left.\left\langle\hat{\sigma}_{+}(t) \hat{\sigma}_{-}(t)\right\rangle=\left\langle\hat{\sigma}_{z}(t)\right\rangle+1\right) / 2$

together with Eq. (44), the probability for the twolevel atom to be on the upper level is found to be

$\rho_{a a}(t)=\rho_{a a}(0) e^{-\Gamma t}+\frac{\Gamma-\xi}{2 \Gamma}\left(1-e^{-\Gamma t}\right)$,

where $\rho_{a a}(t)=\left\langle\hat{\sigma}_{+}(t) \hat{\sigma}_{-}(t)\right\rangle$ is the probability for the atom to be on the upper level. If the atom is initially on the upper level, then $\rho_{a a}(0)=1$. Hence, one can write Eq. (48) in the form

$\rho_{a a}(t)=\frac{e^{-\Gamma t}}{1+4 \varepsilon^{2} / \kappa^{2}}+\frac{4 \varepsilon^{2} / \kappa^{2}}{1+4 \varepsilon^{2} / \kappa^{2}}$,

which takes, in a steady state, the form

$\rho_{a a}(t)=\frac{4 \varepsilon^{2} / \kappa^{2}}{1+4 \varepsilon^{2} / \kappa^{2}}$.

It can also be seen from Fig. 5 that the probability for the atom to be on the upper level decays exponentially in the absence of a parametric amplifier and approaches to zero in a steady state. However, in the presence of a parametric amplifier, the probability for the atom to be on the upper level is different from zero. This is because there are photons in the cavity that can be absorbed by the atom.

\section{Quadrature Variances} and Mean Photon Number

It is a well-established matter that the squeezing properties of a single-mode cavity radiation can be described in terms of the quadrature operators

$\hat{a}_{+}=\hat{a}^{\dagger}+\hat{a}$

and

$\hat{a}_{-}=i\left(\hat{a}^{\dagger}-\hat{a}\right)$.

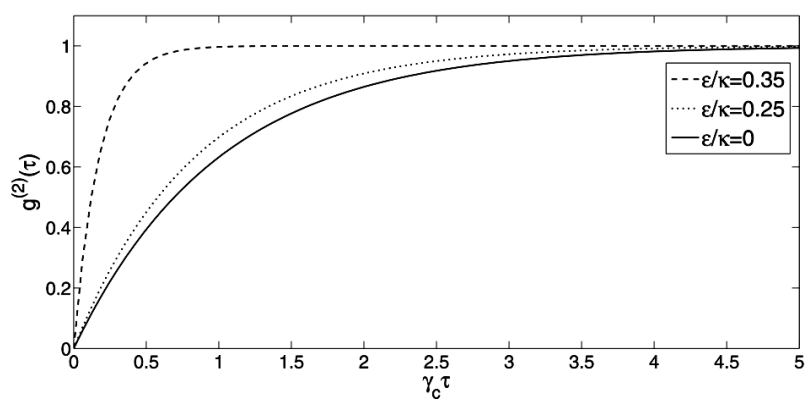

Fig. 4. Plots of the second-order correlation function (Eq. (46)) versus $\gamma_{c} \tau$ for different values of $\varepsilon / \kappa$

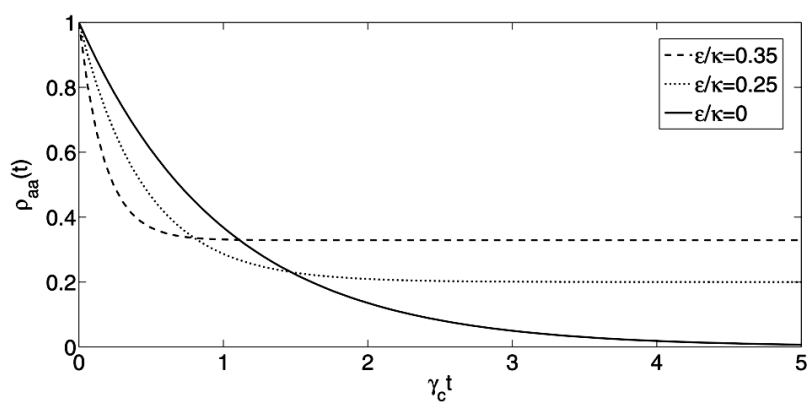

Fig. 5. Plots of the probability for the two-level atom to be on the upper level (Eq. (49)) versus $\gamma_{c} t$ for different values of $\varepsilon / \kappa$

These quadrature operators satisfy the commutation relation $\left[\hat{a}_{+}, \hat{a}_{-}\right]=2 i$. On the basis of these definitions, a single-mode light is said to be in a squeezed state, if either $\Delta a_{+}^{2}<1$ or $\Delta a_{-}^{2}<1$. Furthermore, one can show with the use of Eq. (10) along with the results $\left\langle\hat{\sigma}_{+}(t)\right\rangle=\left\langle\hat{\sigma}_{-}(t)\right\rangle=0$ that $\left\langle\hat{a}^{\dagger}\right\rangle=\langle\hat{a}\rangle=0$. The variances of the quadrature operators can be put in the normal order in the form

$\left(\Delta \hat{a}_{ \pm}\right)^{2}=1+2\left\langle\hat{a}^{\dagger} \hat{a}\right\rangle \pm\left[\left\langle\hat{a}^{2}\right\rangle+\left\langle\hat{a}^{\dagger 2}\right\rangle\right]$.

With the aid of Eqs. (8) and (9), it is possible to see that

$$
\begin{aligned}
& \left\langle\hat{a}^{2}(t)\right\rangle_{s s}=\frac{\varepsilon / \kappa}{1-4 \varepsilon^{2} / \kappa^{2}}-\frac{2 \gamma_{c} \varepsilon / \kappa^{2}}{\left(1-4 \varepsilon^{2} / \kappa^{2}\right)^{2}\left(1+4 \varepsilon^{2} / \kappa^{2}\right)}+ \\
& +\frac{\gamma_{c} \varepsilon^{2} / \kappa^{2}}{\left(1-4 \varepsilon^{2} / \kappa^{2}\right)^{2}}, \\
& \left\langle\hat{a}^{\dagger}(t) \hat{a}(t)\right\rangle_{s s}=\frac{2 \varepsilon^{2}}{\kappa^{2}-4 \varepsilon^{2}}-\frac{4 \gamma_{c} \varepsilon^{2} / \kappa^{3}}{\left(1-4 \varepsilon^{2} / \kappa^{2}\right)^{2}\left(1+4 \varepsilon^{2} / \kappa^{2}\right)}+ \\
& +\frac{2 \gamma_{c} \varepsilon^{2} / \kappa^{3}}{\left(1-4 \varepsilon^{2} / \kappa^{2}\right)^{2}} .
\end{aligned}
$$

It is clear that the first term in Eq. (55) represents the mean photon number of the signal light, when 


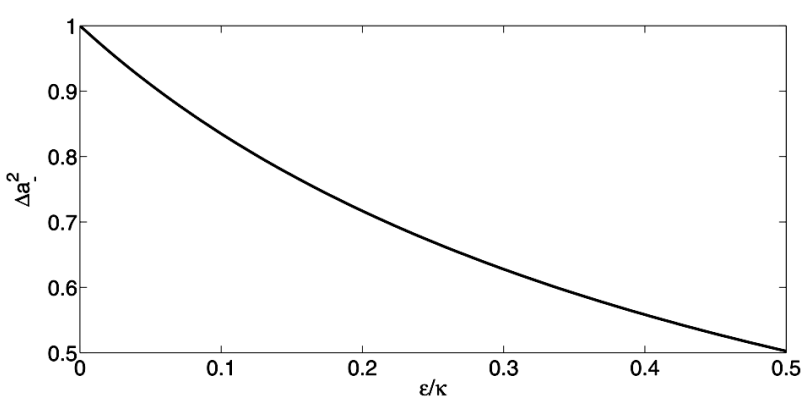

Fig. 6. Plot of the the quadrature variance of cavity radiation (Eq. (59)) versus $\varepsilon / \kappa$ for $\gamma_{c} / \kappa=0.01$

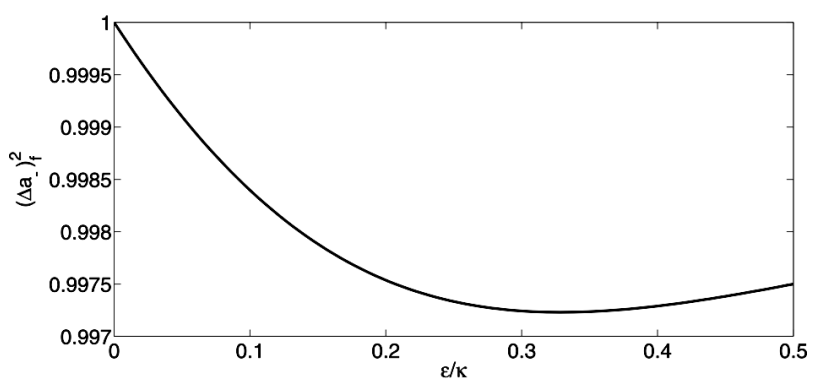

Fig. 7. Plot of the quadrature variance of the fluorescent light (Eq. (60)) versus $\varepsilon / \kappa$ for $\gamma_{c} / \kappa=0.01$

$\gamma_{c}=0$, whereas the second term gives the mean number of absorbed signal photons, and the remaining one represents the mean number of photons emitted by the two-level atom (when $\gamma_{c} \neq 0$ ). Adding the last two terms, we see that

$\bar{n}=\frac{2 \varepsilon^{2}}{\kappa^{2}-4 \varepsilon^{2}}-\frac{2 \gamma_{c} \varepsilon^{2} \kappa}{\left(\kappa^{2}-4 \varepsilon^{2}\right)\left(\kappa^{2}+4 \varepsilon^{2}\right)}$.

In this result, the second term is negative due to a higher mean number of photons absorbed by the two-level atom as compared with the mean number of photons emitted by the atom. From whence, we can find the mean number of of photons emitted by the two-level atom (called fluorescent light). This is described as

$\bar{n}_{f}=\frac{2 \gamma_{c} \varepsilon^{2} / \kappa^{3}}{\left(1-4 \varepsilon^{2} / \kappa^{2}\right)^{2}}$.

Now in view of Eq. (54), the quadrature variance takes the form

$\left(\Delta \hat{a}_{ \pm}\right)^{2}=1 \pm \frac{\kappa}{\varepsilon}\left(1 \pm \frac{2 \varepsilon}{\kappa}\right)\left\langle\hat{a}^{\dagger} \hat{a}\right\rangle$.
Taking Eq. (55) into account, the quadrature variance of the cavity radiation can be written as

$$
\left(\Delta \hat{a}_{ \pm}\right)^{2}=1 \pm \frac{2 \varepsilon / \kappa\left(1-\gamma_{c} / \kappa\right)+8 \varepsilon^{3} / \kappa^{3}}{(1 \mp 2 \varepsilon / \kappa)\left(1+4 \varepsilon^{2} / \kappa^{2}\right)} .
$$

We note that the cavity mode is in a squeezed state, and the squeezing occurs in the minus quadrature. In Fig. 6, the plot indicates that the cavity mode is in a squeezed state, and the degree of squeezing increases in the presence of a parametric amplifier. A highly correlated pairs of photons of a signal light are responsible for the squeezing of this light. A decrease in the degree of squeezing of the signal light is due to the loss of correlations between signal photon pairs, when the atom absorbs a single photon.

The quadrature variances of fluorescent light is found to be

$\left(\Delta \hat{a}_{ \pm}\right)_{f}^{2}=1 \pm \frac{2 \gamma_{c} \varepsilon / \kappa^{2}}{(1 \mp 2 \varepsilon / \kappa)\left(1-4 \varepsilon^{2} / \kappa^{2}\right)}$.

From expression (60), we conclude that the fluorescent light is in a squeezed state, and the squeezing occurs in the minus quadrature. Figure 7 indicates that the degree of squeezing of the fluorescent light is very small.

\section{Conclusion}

The quantum properties of a two-level atom inside a degenerate parametric oscillator, when the cavity is coupled to a vacuum reservoir, are thoroughly analyzed. Employing the master equation for the system under consideration and the quantum Langevin equation, the time evolution of the expectation values of the cavity mode and atomic operators in the badcavity limit are obtained. Moreover, using these results, the power spectrum and the second-order correlation function of the fluorescent light emitted by the two-level atom are obtained. In addition, the mean photon number and the quadrature squeezing for the cavity mode and for fluorescent light are determined.

It is found that the presence of a parametric amplifier leads to an increase in the width of the power spectrum of the fluorescent light. On the other hand, the presence of a parametric amplifier decreases the width of the power spectrum of the cavity mode. In addition, the photons in the fluorescent light are antibunched, as always the case. The effect of the presence of a parametric amplifier on the second-order 
correlation function is to enhance its decay rate. Furthermore, the presence of a parametric amplifier enhances the probability for the atom to be on the upper level. It is observed that the cavity mode is in a squeezed state, and the squeezing occurs in the minus quadrature.

1. C.W. Gardiner. Inhibition of atomic phase decays by squeezed light: A direct effect of squeezing. Phys. Rev. Lett. 56, 1917 (1986).

2. H.J. Carmichael, A.S. Lane, D.F. Walls. Resonance fluorescence from an atom in a squeezed vacuum. Phys. Rev. Lett. 58, 2539 (1987).

3. H.J. Carmichael, A.S. Lane, D.F. Walls. Resonance fluorescence in a squeezed vacuum. J. Mod. Opt. 34, 821 (1987).

4. R. Vyas, S. Singh. Resonance fluorescence with squeezedlight excitation. Phys. Rev. A 45, 8095 (1992).

5. A.S. Parkins. Rabi sideband narrowing via strongly driven resonance fluorescence in a narrow-bandwidth squeezed vacuum. Phys. Rev. A 42, 4352 (1990).

6. J.I. Cirac, L.L. Sanchez-Soto. Suppression of spontaneous emission by squeezed light in a cavity. Phys. Rev. A 44, 1948 (1991).

7. J.I. Cirac. Interaction of a two-level atom with a cavity mode in the bad-cavity limit. Phys. Rev. A 46, 4354 (1992).

8. P.R. Rice, L.M. Pedrotti. Fluorescent spectrum of a single atom in a cavity with injected squeezed vacuum. J. Opt. Soc. Am. B 9, 2008 (1992).

9. P.R. Rice, C.A. Baird. Interaction of a two-level atom with a squeezed vacuum: Photon statistics and spectra. Phys. Rev. A 53, 3633 (1996).

10. W.S. Smyth, S. Swain. Anomalous resonance fluorescence from an atom in a cavity with injected squeezed vacuum. Phys. Rev. A 53, 2846 (1996).

11. D. Erenso, R. Vyas. Two-level atom coupled to a squeezed vacuum inside a coherently driven cavity. Phys. Rev. A 65 063808 (2002).
12. G.S. Agarwal. Effects of optical gain and cavity-mode squeezing on the Mollow spectrum. Phys. Rev. A 40, 4138 (1989).

13. S. Jin, M. Xiao. Extra intracavity squeezing of a degenerate optical parametric oscillator coupling with $N$ two-level atoms. Phys. Rev. A 49, 499 (1994).

14. E. Alebachew. A coherently driven two-level atom inside a parametric oscillator. J. Mod. Opt. 55, 1159 (2008).

15. Geoffery New. Introduction to Nonlinear Optics (Cambridge Univ. Press, 2011).

16. Fesseha Kassahun, Fundamental of Quantum Optics (Lulu, 2008).

Received 27.02.18

T. Абебе, Н. Гемечу

ДВОРІВНЕВИЙ АТОМ

У СТИСЛОМУ ВИПРОМІНЮВАННІ ОПТИЧНИХ

ПАРАМЕТРИЧНИХ ОСЦИЛЯТОРІВ

$\mathrm{P}$ е $з$ ю м е

Аналізується динаміка когерентно збудженого дворівневого атома, пов'язаного з параметричним підсилювачем і вакуумним резервуаром. Використовуючи керуюче рівняння і квантове рівняння Ланжевена, вивчаються квантові властивості світла і тимчасова еволюція очікувань для порожнинної моди світла і операторів, що характеризують атом. На основі цих результатів, кореляційних властивостей операторів шумів і наближення великих часів, розраховані середнє число фотонів, спектр потужності, кореляційна функція другого порядку і квадратичні дисперсії для флуоресценції і для порожнинної моди світла. Показано, що напівширина спектра потужності флуоресценції зростає за наявності параметричного підсилювача і зменшується для порожнинної моди світла. Розрахована ймовірність для атома бути на верхньому рівні за наявності параметричного підсилювача. 